\title{
$\mathrm{HS}-\mathrm{SPME} \mathrm{GC} / \mathrm{MS}$ 를 이용한 젤펜잉크의 휘발성 성분
}

\author{
김창성 · 최미정 ${ }^{1} \cdot$ 선일식 · 박성우 ${ }^{1, \star}$ \\ 한국화학시험연구원, 충남대학교 과학수사학과 \\ (2010. 6. 18. 접수, 2010. 7. 28. 승인)
}

\section{Volatile organic components of gel pen inks by HS-SPME GC/MS}

\author{
Chang-Seong Kim, Mi-Jung Choi ${ }^{1}$, Yale-Shik Sun and Sung-Woo Park ${ }^{1, \star}$ \\ Hazard Evaluation Center, KoreaTesting \& Research Institute, Gimpo, Gyunggi-Do, 415-871, Korea \\ ${ }^{I}$ Dept. of Scientific Criminal Investigation, Chungnam National University Daejeon, 305-764, Korea \\ (Received June 18, 2010; Accepted July 28, 2010)
}

\begin{abstract}
요 약: 젤펜잉크 중 휘발성 성분을 스크리닝하여 개체특성자료를 확보하고자 3 개국에서 생산된 16 종의 흑색 젤펜잉크 카트리지를 시료로 분석하였다. 검출된 성분은 총 20 종으로 isopropylalcohol, 2-methyl-2propanol, 2-butanone, hydrazinecarbothioamide, benzeneacetic acid(ethyl ester), benzeneacetic acid, dimethoxymethy-silane, 2,2-dimethoxybutane, tetrahydro-2-methyl-furan, 1,2-ethanediol, silicic acid (tetramethyl ester), 1,2-propanediol, propyleneglycol, 3-ethyl-3-hexanol, 1,1-dipropoxy-propane, 2-butoxy-ethanol, 2,2'oxybisethanol, 1-butyl-benzene, 2-pyrrolidinone, 2-(2-butoxyethoxy)-ethanol이었다. 그중 2,2-dimethoxybutane 은 3.02 47\%의 비율범위로 16 종 젤펜 모든 시료에서 검출되었으며 tetrahydro-2-methyl-furan의 경우 $1.19 \sim 52.19 \%$ 로 GP1을 제외한 15 종의 시료에서 검출되었다. 특히 일본산 제품 5 종에서는 1,2-ethanediol가 52.83 95.84\%의 범위, 한국산 동아제품 3종에서는 76.17 93.51\%의 범위로 1,2-propanediol가 높은 함유율 로 나타나 생산국에 따른 특성과 제조사별 특정성분의 함유분포를 알 수 있어서 잉크 휘발성 성분으로 개체특성을 확인하였다.
\end{abstract}

\begin{abstract}
In this study, VOCs of black gel pen inks was screening to identify individual characteristic of gel pen inks. Detected VOCs was total 20 species(isopropylalcohol, 2-methyl-2-propanol, 2-butanone, hydrazinecarbothioamide, benzeneacetic acid (ethyl ester), benzeneacetic acid, dimethoxymethy-silane, 2,2-dimethoxybutane, tetrahydro-2-methyl-furan, 1,2-ethanediol, silicic acid (tetramethyl ester), 1,2-propanediol, propyleneglycol, 3-ethyl3-hexanol, 1,1-dipropoxy-propane, 2-butoxy-ethanol, 2,2'-oxybisethanol, 1-butyl-benzene, 2-pyrrolidinone, 2-(2butoxyethoxy)-ethanol). We detected 2,2-dimethoxybutane (3.02 47\% ratio) and tetrahydro-2-methyl-furan (1.19 52.19\% ratio), 1,2-ethanediol (52.83 $95.84 \%$ ratio). In case of manufacturer, manufactured ink was able to discriminate between Japan and Korea by distinct characteristics (Japan: 1,2-ethanediol, 52.83 95.84\%, Korea: 1,2-propanediol, 76.17 $93.51 \%$ ). The results of this study indicated that distinct characteristic about manufacturers and brands could make a classifring tool of inks for identification of between gel pen inks.
\end{abstract}

Key words: forensic science, gel pen inks, classification, discrimination, HS-SPME GC/MS

Corresponding author

Phone : +82-(0)42-821-5240 Fax : +82-(0)42-822-5236

E-mail : swpark05@cnu.ac.kr 


\section{1. 서 론}

필기구류의 특성 파악은 위조수표, 어음, 계약서, 유언장과 같은 문제시되는 문서증거물에 사용된 필 기구가 동일한 것인지를 확인하기 위해 형태적 또는 성분 특성의 파악은 중요하다. ${ }^{1,2}$ 문서위에 기재된 필 기구의 식별방법 중 비파괴적인 방법으로는 현미경을 이용하여 획의 이동방향을 알 수 있는 줄무늬 흔적인 찰흔(striation)과 잉크의 과도한 흐름으로 인하여 종이 위에 남게 되는 뭉친 잉크흔적(gooping) 또는 젤펜에서 나타나는 잉크 획의 가장자리로의 이동과 같은 기재후 나타나는 형태적인 특성이나 SEM(scanning electron microscopy) $)^{3-5}$ 을 이용하여 발색성분인 염료(dye) 또는 안료(pigment)의 분포형태로 확인한다. 또한 SERRS (surface-enhanced resonance raman spectroscopy), ${ }^{6-8}$ FT-IR spectroscopy ${ }^{9}$ 등의 광학적 분석이나 $\mathrm{GC}$ (gas chromatography), LC (liquid chromatography)를 이용 한 잉크성분 중 전달물질(vehicles)로 사용되는 휘발 성 용매나 crystal violet, methyl violet, victoria blue 등의 주요 염료를 분석하여 필기구에 포함된 잉크의 개별특성을 확인하고 있다.

필기구로 사용되고 있는 펜에는 잉크의 구성성분 특성 및 필기펜촉의 종류에 따라 만년 필(fountain pen), 볼펜(ballpoint pen), 중성펜 혹은 젤펜(gel pen), 펠트펜(fiber tip pen) 등으로 구분되어 사용되 고 있다. ${ }^{10}$ 그 중 젤펜은 부드러운 움직임, 선명한 색 상과 자연친화적인 특성 등의 장점으로 개발되어 그 사용이 증가하고 있는 추세의 펜으로써 필기시에 볼 펜의 단점인 찰흔, 뭉친 잉크흔적이 없고 잉크의 분 배감이 양호하여 사용량이 증가하고 있는 필기구이 다. 젤펜 잉크의 주성분과 발색성분으로는 염료 또 는 안료를 사용하고 전달물질로는 물, 수지, 비이온 성 계면활성제 등으로 구성되어 있다고 알려져 있다 ${ }^{11}$. 특히 발색성분으로 안료 형태를 사용하는 점이 볼펜 과의 차이점으로 이와 같은 특징은 선행연구인 $\mathrm{SEM}$ 을 이용하여 3 개국에서 생산된 16 종 젤펜의 잉 크 형태 특성을 확인한 연구에서 염료와 안료의 형 태적 특성을 파악하여 개체식별을 위한 자료를 확보 한 바 있다. 이에 본 연구에서는 3 개국에서 생산된 16 종의 흑색 젤펜잉크 카트리지를 시료로 휘발성 성 분을 스크리닝하여 개체특성과 시료간의 군집성 자 료를 확보하여 식별자료로 활용할 수 있는지를 확인 하고자 실시하였다.

\section{2. 실 험}

\section{1. 시료 및 기구}

젤펜의 시료는 시중에서 판매되고 있는 것으로 3 개 국가에서 생산된 한국 9 종, 일본 5 종, 독일 2 종으 로 $0.7 \mathrm{~mm}$ 의 medium 크기의 point를 보유한 총 16 종을 구입하였으며 시료목록은 Table 1과 같다.

젤펜 카트리지 잉크의 volatile organic component (VOC)를 분석하기 위하여 GC/MS (Simadzu 2010, Japan), SPME headspace sampler는 $75 \mu \mathrm{m}$ Carboxen $^{\mathrm{TM}}$ polydimethylsiloxane (PDMS)를 사용하였다.

\section{2. 실험방법}

2.2.1. HS-SPME GC/MS에 의한 잉크 카트리지 VOC 분석

잉크 카트리지를 메탄올에 10 배 희석하여 $100 \mu \mathrm{L}$ 를 conical vial (Supelco ${ }^{\mathrm{TM}}$, Buchs, Switzerland)에 주 입하고 20 분간, $90^{\circ} \mathrm{C}$ 에서 SPME $75 \mu \mathrm{m}$ Carboxen ${ }^{\mathrm{TM}}$ polydimethylsiloxane(PDMS)를 사용하여 수집하고 분 석하였다(Table 2).

\subsection{2. 통계처리법}

실험의 결과는 SAS 통계 프로그램인 JMP package (SAS statistical discovery ${ }^{\mathrm{TM}}$ )를 이용하여 시료 간의 군집분포성을 조사하였다. 즉 측정한 변수를 이용하 여 시료 간의 유사성을 거리로 표현하거나 비유사성

Table 1. List of black gel pens

\begin{tabular}{clll}
\hline \hline Sample No. & Country & Manufacture & \multicolumn{1}{c}{ Pen name } \\
\hline GP 1 & Korea & Dong-A & Jellzone \\
GP 2 & & & 3-Zero \\
GP 3 & & & Miffy \\
GP 4 & & Jellitto \\
GP 5 & & MonAmi & N.Jell \\
GP 6 & & & Jell-Line \\
GP 7 & & & Evergreen.265 \\
GP 8 & & & Tixjell \\
GP 9 & & & JELLER502 \\
GP 10 & Japan & Pentel & ENERGEL \\
GP 11 & & & HybridGelGsipFine \\
GP 12 & & & Hybrid Gel Gsip Dx \\
GP 13 & & ZEBRA & SARASA \\
GP 14 & & SAKURA & GELLY ROLL FINE \\
GP 15 & Germany & STAEDTLER triplusgel-liner \\
GP 16 & & & Gelion \\
\hline
\end{tabular}


Table 2. Analytical conditions of HS-SPME GC/MS for determination of VOCs in black gel pen ink catridge

\begin{tabular}{ll}
\hline \hline & GC Info. Simadzu 2010 \\
\hline Column & DB-624 $(60 \mathrm{~m} \times 0.32 \mathrm{mml} . \mathrm{D}$, \\
& $1.8 \mu \mathrm{mdf})$ \\
Oven Program & $50{ }^{\circ} \mathrm{C}(3 \mathrm{~min})-10{ }^{\circ} \mathrm{C} / \mathrm{min}-250{ }^{\circ} \mathrm{C}$ \\
Inlet Temp. & $(5 \mathrm{~min})$ \\
Carrier Gas & $230{ }^{\circ} \mathrm{C}$ \\
Inlet Mode & He at a constant flow of $1 \mathrm{~mL} / \mathrm{min}$ \\
\hline Detector MSD(EI) & split $(25: 1)$ \\
\hline Mass range & $35 \sim 500$ amu \\
Acquisition Rate & $20 \mathrm{spectra} / \mathrm{sec}$ \\
Ionization energy & $70 \mathrm{eV}$ \\
Ion Source Temperature & $230{ }^{\circ} \mathrm{C}$ \\
\hline SPME Headspace sampler \\
\hline SPME fiber & $75 \mu \mathrm{m} \mathrm{Carboxen}{ }^{\mathrm{TM}}-$ polydimethyl- \\
Extraction time & siloxane(PDMS) \\
Extraction Temperature & $90{ }^{\circ} \mathrm{C}$ \\
Sample volume & $100 \mu \mathrm{L}$ \\
\hline
\end{tabular}

으로 계산해내는 multivariate method중 hierarical cluster 를 이용하고 계층적 군집 중 WARD법을 사용하였으 며 two way clustering(다변량군집 분석)을 시도하여 군집성에 대한 기초자료를 파악하였다. 또한 시료에
서 검출된 성분중에서 개별특성을 규정지을 수 있는 성분을 찾기 위하여 주성분분석을 이용하여 조사하 였다.

\section{3. 결과 및 고찰}

\section{1. 젤펜 잉크 카트리지 $\mathrm{VOC}$ 분석}

잉크 카트리지의 $\mathrm{VOC}$ 를 검출하기 위하여 HS$\mathrm{SPME} \mathrm{GC/MS}$ 를 이용한 시료에서의 이온크로마토그 램이다(Fig. 1).

\section{2. 잉크 카트리지 휘발성성분}

$\mathrm{HS}-\mathrm{SPME} \mathrm{GC/MS}$ 를 이용하여 휘발성성분을 검출한 결과는 Table 3 이며 검출된 성분으로는 isopropylalcohol, 2-methyl-2-propanol, 2-butanone, hydrazinecarbothioamide, benzeneacetic acid (ethyl ester), benzeneacetic acid, dimethoxymethy-silane, 2,2-dimethoxybutane, tetrahydro-2-methyl-furan, 1,2-ethanediol, silicic acid (tetramethyl ester), 1,2-propanediol, propyleneglycol, 3ethyl-3-hexanol, 1,1-dipropoxy-propane, 2-butoxy-ethanol, 2,2'-oxybisethanol, 1-butyl-benzene, 2-pyrrolidinone, 2(2-butoxyethoxy)-ethanol으로 총 20종이 검출되었다 (Table 3).

그중 2,2-dimethoxybutane의 경우 $3.02 \sim 47 \%$ 의 범

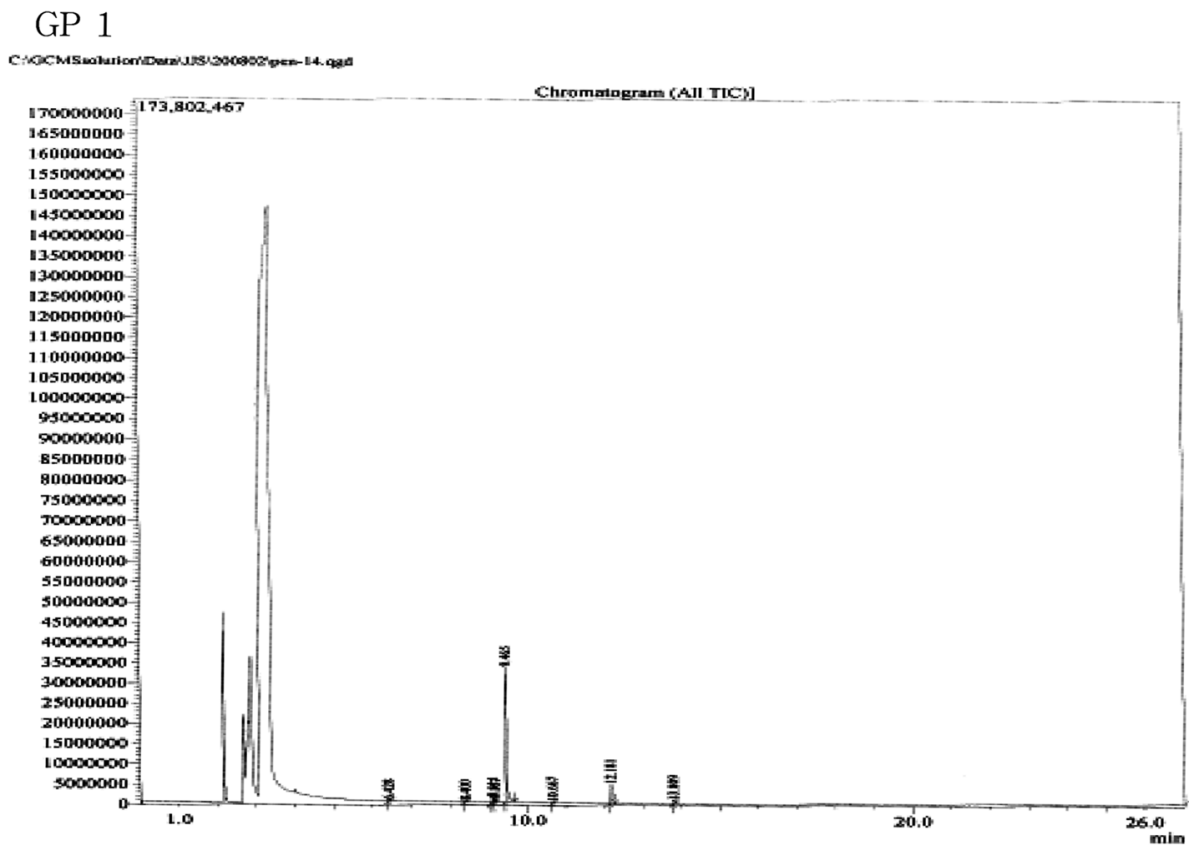

Fig. 1. Total ion chromatogram of VOCs obtained by HP-SPME GC/MS. 
Table 3. The identified VOCs in black gel pen ink catridge

\begin{tabular}{|c|c|c|c|c|c|c|c|c|c|c|c|c|c|c|c|c|}
\hline \multirow[b]{3}{*}{ Component } & \multicolumn{9}{|c|}{ Korea } & \multicolumn{5}{|c|}{ Japan } & \multirow{2}{*}{\multicolumn{2}{|c|}{$\begin{array}{l}\text { Germany } \\
\text { staedtler }\end{array}$}} \\
\hline & \multicolumn{4}{|c|}{ Dong-A } & \multicolumn{5}{|c|}{ MonAmi } & \multicolumn{3}{|c|}{ Pentel } & \multicolumn{2}{|c|}{ zebra sakura } & & \\
\hline & GP1 & GP2 & GP3 & GP4 & GP5 & GP6 & GP7 & GP8 & GP9 & GP10 & GP11 & GP12 & GP13 & GP14 & GP15 & GP16 \\
\hline Isopropylalcohol & 9.31 & - & - & - & - & - & - & - & - & - & - & - & - & - & - & - \\
\hline 2-Methyl-2-propanol & - & - & - & - & - & - & - & - & - & - & - & - & - & - & 2.44 & - \\
\hline 2-Butanone & - & 2.22 & 0.66 & 0.83 & 9.92 & - & 9.68 & 5.36 & 4.23 & - & - & 0.29 & 2.02 & 0.6 & 0.72 & 9.43 \\
\hline Hydrazinecarbothioamide & - & 2.43 & - & - & - & - & 11.59 & - & - & - & - & - & - & - & 0.73 & 7.24 \\
\hline $\begin{array}{l}\text { Benzeneacetic acid } \\
\quad \text { (ethyl ester) }\end{array}$ & - & - & - & 0.83 & 8.36 & - & - & - & 3.96 & - & - & - & - & - & - & - \\
\hline Benzeneacetic acid & - & - & - & - & - & - & - & - & - & - & - & - & - & 0.75 & - & - \\
\hline Dimethoxymethyl-silane & - & - & - & - & - & - & - & 5.5 & - & - & - & - & - & - & - & - \\
\hline 2,2-Dimethoxybutane & 20.95 & 10.04 & 3.2 & 4.15 & 34.48 & 47.81 & 41.27 & 18.79 & 15.14 & 19.5 & 14.7 & 1.27 & 8.95 & 3.02 & 3.02 & 13.68 \\
\hline Tetrahydro-2-methyl-furan & - & 2.21 & 1.53 & 1.19 & 18.86 & 52.19 & 22.82 & 13.68 & 13.39 & 27.67 & 22.56 & 1.26 & 7.75 & 1.74 & 1.6 & 19.62 \\
\hline 1,2-Ethanediol & - & - & - & - & 1.21 & - & - & 15.55 & 20.26 & 52.83 & 62.73 & 95.84 & 81.28 & 80.78 & 10.06 & 12.38 \\
\hline $\begin{array}{l}\text { Silicic acid } \\
\quad \text { (tetramethyl ester) }\end{array}$ & - & 2.73 & 1.1 & 1.08 & 18.17 & - & 14.63 & 11.42 & 12.99 & - & - & - & - & - & 3.05 & - \\
\hline 1,2-Propanediol & - & 80.37 & 93.51 & 76.17 & - & - & - & - & - & - & - & - & - & - & 78.37 & 37.65 \\
\hline Propyleneglycol & 69.74 & - & - & - & - & - & - & - & - & - & - & - & - & - & - & - \\
\hline 3-Ethyl-3-hexanol & - & - & - & 0.7 & - & - & - & - & - & - & - & - & - & - & - & - \\
\hline 1,1-Dipropoxy-propane & - & - & - & - & - & - & - & - & - & - & - & - & - & 0.33 & - & - \\
\hline 2-Butoxy-ethanol & - & - & - & - & - & - & - & - & - & - & - & - & - & 10.76 & - & - \\
\hline 2,2'-Oxybisethanol & - & - & - & - & - & - & - & 20.33 & 21.76 & - & - & 1.01 & - & 2.02 & - & - \\
\hline 1-Butyl-benzene & - & - & - & - & - & - & - & - & - & - & - & 0.35 & - & - & - & - \\
\hline 2-Pyrrolidinone & - & - & - & 15.06 & - & - & - & - & - & - & - & - & - & - & - & - \\
\hline 2-(2-Butoxyethoxy)-ethanol & $1-$ & - & - & - & - & - & - & 9.38 & 8.27 & - & - & - & - & - & - & - \\
\hline Total $(\%)$ & 100 & 100 & 100 & 100 & 100 & 100 & 100 & 100 & 100 & 100 & 100 & 100 & 100 & 100 & 100 & 100 \\
\hline
\end{tabular}

위로 16 종 젤펜 모든 시료에서 검출되었으며 tetrahydro-2-methyl-furan의 경 우 1.19 52.19\%로 GP1을 제외한 15 종의 시료에서 검출되었다. 2-butanone의 경우 0.29 9.92\%의 범위로 GP1, GP6의 한국산 시료 와 GP10, GP11의 일본산 시료에서만 검출되지 않았 으며 1,2-ethanediol의 경우 10 종의 시료에서 검출되 었으며 한국산 시료 동아제품인 GP1, GP2, GP3, $\mathrm{GP} 4$ 에서는 검출되지 않았으나 모나미제품 5종 중 3 종(GP5, GP8, GP9)에서 검출되는 특성을 확인할 수 있었다. 그러나 일본산 제품 5종(GP10, GP11, GP12, $\mathrm{GP} 13, \mathrm{GP} 14)$ 과 독일산 2종(GP15, GP16)에서는 모두 검출되고 특히 일본산의 경우 $52.83 \sim 95.84 \%$ 의 범위 로 높은 비율을 차지함을 알 수 있었다. silicic acid (tetramethyl ester)의 경우 $1.1 \sim 18.17 \%$ 의 범위로 한국 산 9종 중 7종 $(\mathrm{GP} 2, \mathrm{GP} 3, \mathrm{GP} 4, \mathrm{GP} 5, \mathrm{GP} 7, \mathrm{GP} 8$, $\mathrm{GP} 9)$ 에서 검출되었으며 독일산 1 종(GP15)에서 검출 되었으며 일본산에서는 검출되지 않았다. 1,2propanediol의 경우, 한국산 동아제품 3종 $(\mathrm{GP} 2, \mathrm{GP} 3$,
$\mathrm{GP} 4)$ 과 독일산 2종(GP15, GP16)에서 검출되었으며 특히 한국산 동아제품의 경우 76.17 93.51\%의 범위 로 높은 함유율을 확인할 수 있었다. 또한 한국산 $\mathrm{GP} 1$ 에서는 propyleneglycol이 $69.74 \%$ 로 검출되고 isopropylalcohol이 $9.31 \%$ 로 검출되고 다른시료에서는 검출되지 않았다. 2-pyrrolidinone의 경우 한국산 시료 $\mathrm{GP} 4$ 에서만 $15.06 \%$ 로 검출되고 2-(2-butoxyethoxy)ethanol은 한국산 GP8과 GP9의 모나미제품에서만 검 출되었다. 일본산 sakura제품의 경우 다른시료에서는 검출되지 않는 1,1-dipropoxy-propane과 2-butoxyethanol이 검출되는 것을 확인할 수 있었다.

\section{3. 통계분석을 이용한 군집 및 개별특성}

젤펜 잉크 카트리지 시료의 휘발성성분 분석치를 대입하여 시료간의 유사성과 비유사성을 거리로 표 현하여 다량의 시료에서 검출된 성분의 유무나 분석 치간의 상호상관성이 함께 표현되어지는 two way clustering을 시도하였다. 
Table 4. Clustering history of the relationship between gel pen groups and detected VOCs

\begin{tabular}{|c|c|c|c|c|}
\hline Components $\quad$ Cluster & Group 1 & Group 2 & Group 3 & Group 4 \\
\hline Isopropylalcohol & 9.31 & - & - & - \\
\hline 2-Methyl-2-propanol & - & 0.61 & 0.41 & - \\
\hline 2-Butanone & - & 1.11 & 4.42 & 0.58 \\
\hline Hydrazinecarbothioamide & - & 0.79 & 2.05 & - \\
\hline Benzeneacetic acid(ethyl ester) & - & 0.21 & 1.53 & - \\
\hline Benzeneacetic acid & - & - & - & 0.15 \\
\hline dimethoxymethy-silane, & - & - & 0.92 & - \\
\hline 2,2-dimethoxybutane & 20.95 & 5.10 & 24.92 & 9.49 \\
\hline Tetrahydro-2-methyl-furan & - & 1.63 & 18.39 & 12.20 \\
\hline 1,2-Ethanediol & - & 2.52 & 5.97 & 74.69 \\
\hline Silicic acid(tetramethyl ester) & - & 1.99 & 8.06 & - \\
\hline 1,2-propanediol & - & 82.11 & 25.76 & - \\
\hline Propyleneglycol & 69.74 & - & - & - \\
\hline 3-ethyl-3-hexanol & - & 0.18 & 0.12 & - \\
\hline 1,1-dipropoxy-propane & - & - & - & 0.07 \\
\hline 2-butoxy-ethanol, & - & - & - & 2.15 \\
\hline 2,2'-oxybisethanol & - & - & 3.39 & 0.61 \\
\hline 1-Butyl-benzene & - & - & - & 0.07 \\
\hline 2-Pyrrolidinone & - & 3.77 & 2.51 & - \\
\hline 2-(2-Butoxyethoxy)-ethanol & - & - & 1.56 & - \\
\hline
\end{tabular}

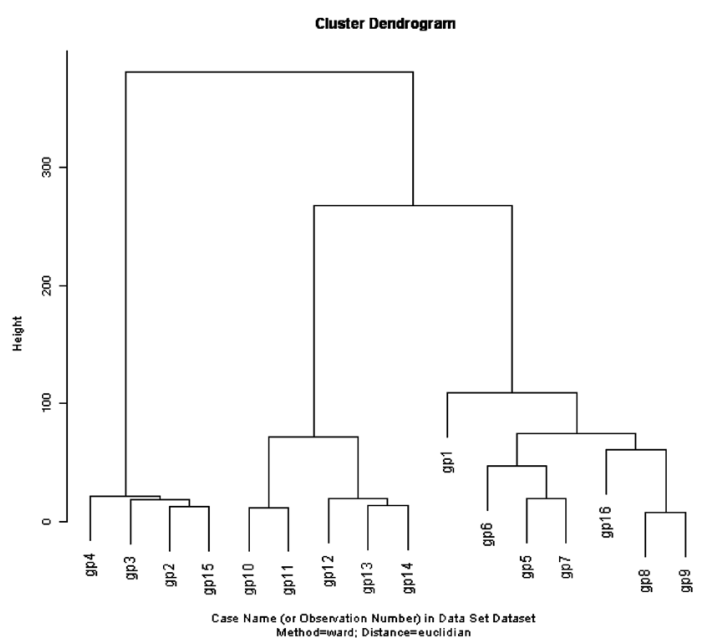

Fig. 2. Dendrogram showing the relationship between gel pen groups and detected VOCs using the modified hieradical cluster method (nearest neighbor, euclidean distance).

Table 4는 3개 국가에서 생산된 검은색 젤펜잉크 16 종의 휘발성성분 검출치를 이용한 군집평균값이며 Fig. 2는 군집덴드로그램으로 시료간의 군집성은 4 group으로 구분되어졌다. Group 1은 한국산 동아제품 으로 GP1, group 2는 4종으로 한국산 동아제품인

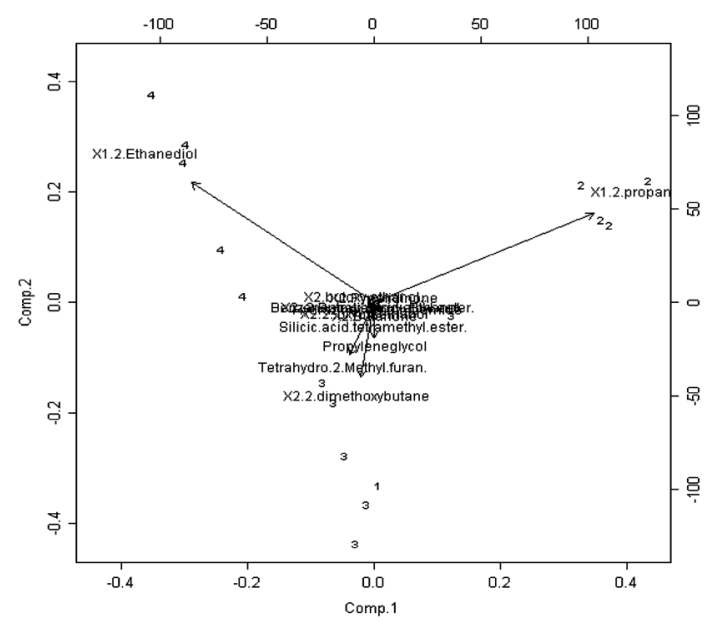

Fig. 3. Clustering history of the relationship between gel pen groups and detected VOCs using PCA analysis.

$\mathrm{GP} 2, \mathrm{GP} 3, \mathrm{GP} 4$ 와 독일산 $\mathrm{GP} 15$ 가 군집을 이루며 group 3은 일본산 모든제품으로 GP10, GP11, GP12, GP13, GP14이며, group 4는 한국산 모나미제품인 $\mathrm{GP} 5, \mathrm{GP} 6, \mathrm{GP7}, \mathrm{GP} 8, \mathrm{GP} 9$ 의 5종과 독일산 GP16 시 료가 포함되어졌다. 또한 주성분 분석을 시도하였을 때 Fig. 3과 같이 군집성을 이룰 수 있는 4 group 각 각의 주요한 성분이 group 1 과 3 의 경우 propy- 
leneglycol, tetrahydro-2-methyl-furan, 2,2-dimethoxybutane이며 group 2는 1,2-propanediol, group 4는 1,2-ethanediol임을 알 수 있었다.

\section{4. 결 론}

젤펜 잉크의 개체식별을 목적으로 3 개 국가에서 생산된 흑색젤펜 16종을 시료로 HS-SPME를 이용하 여 휘발성성분 자료를 확보하여 잉크시료의 개별 특 이성을 확인하여 식별자료로 활용하고자 하였다.

1. 검출된 휘발성성분으로는 isopropylalcohol, 2methyl-2-propanol, 2-butanone, hydrazinecarbothioamide, benzeneacetic acid (ethyl ester), benzeneacetic acid, dimethoxymethy-silane, 2,2-dimethoxybutane, tetrahydro-2-methyl-furan, 1,2-ethanediol, silicic acid (tetramethyl ester), 1,2-propanediol, propyleneglycol, 3-ethyl-3-hexanol, 1,1-dipropoxy-propane, 2-butoxy-ethanol, 2,2'-oxybisethanol, 1-butyl-benzene, 2-pyrrolidinone, 2-(2-butoxyethoxy)-ethanol으로 총 20종이 검출되었다.

그중 2,2-dimethoxybutane은 3.02 47\%의 비율범위 로 16종 젤펜 모든 시료에서 검출되었으며 tetrahydro2-methyl-furan의 경우 1.19 52.19\%로 GP1을 제외한 15 종의 시료에서 검출되었다. 1,2-ethanediol의 경우 일본산 제품 5종 $(\mathrm{GP} 10, \mathrm{GP} 11, \mathrm{GP} 12, \mathrm{GP} 13, \mathrm{GP} 14)$ 과 독일산 2종 $(\mathrm{GP} 15, \mathrm{GP} 16)$ 에서는 모두 검출되고 특 히 일본산의 경우 $52.83 \sim 95.84 \%$ 의 범위로 높은 비율 을 차지함을 알 수 있었으며 1,2-propanediol의 경우, 한국산 동아제품 3 종 $(\mathrm{GP} 2, \mathrm{GP} 3, \mathrm{GP} 4)$ 과 독일산 2 종 (GP15, GP16)에서 검출되었으며 특히 한국산 동아제 품의 경우 76.17 93.51\%의 범위로 높은 함유율을 확 인할 수 있었다. 또한 한국산 GP1에서는 propyleneglycol이 $69.74 \%$ 로 검출되고 isopropylalcohol이 $9.31 \%$ 로 검출되고 다른시료에서는 검출되지 않아 시료별 개체특성을 파악할 수 있었다.

2. 시료간의 군집성에 있어서는 4 group으로 구분 되어, group 1은 한국산 동아제품으로 GP1이 개별특 성이 뛰어났으며 group 2는 4종으로 한국산 동아제
품인 GP2, GP3, GP4와 독일산 GP15, group 3은 일 본산 모든제품인 포함되는 $\mathrm{GP} 10, \mathrm{GP} 11, \mathrm{GP} 12$, GP13, GP14이며, group 4는 한국산 모나미제품인 $\mathrm{GP} 5, \mathrm{GP} 6, \mathrm{GP} 7, \mathrm{GP} 8, \mathrm{GP} 9$ 의 5종과 독일산 $\mathrm{GP} 16$ 시 료가 포함된 군집성을 확인할 수 있었으며 또한 주성 분 분석으로 군집성을 이룰 수 있는 주요한 성분이 group 1과 3의 경우 propyleneglycol, tetrahydro-2-methylfuran, 2,2-dimethoxybutane이며 group 2는 1,2-propanediol, group 4는 1,2-ethanediol임을 알 수 있어서 젤펜의 휘발성 성분의 검출특성으로 개체식별과 제 조사 식별의 가능성을 알 수 있었다.

\section{참고문헌}

1. L. Richard, M. S. Brunelle and R. C. Kenneth, Advances in the forensic analysis and dating of writing ink, Springfield, 13-46(2003).

2. M. N. Gernandt and J. J. Urlaub, J. Forensic Sci., 41, 503-504(1996).

3. Xu Yuanyuan and Wang Jinghan, J. Forensic Sci. Int., 164, 140-143(2006).

4. M. Claybourn and M. Ansell, Sci. Justice, 40, 261271(2000).

5. N. Y. Kim and S. W. Park, Anal. Sci. Tech., 17, 315312(2004)

6. A. Kher, M. Mulholland, E. Green and B. Reedy, Vib. Spectrosc., 40, 270-277(2006).

7. P. C. White, Sci. Justice, 43, 149-152(2003).

8. J. A. Zlotnick and F. P. Smith, J. Chromatogr. 733, 265-272(1999).

9. C. D. Adam and S. L. Sherratt, J. Forensic Sci. Int., 174, 16-25(2008).

10. J. D. Wilson, G. M. Laporte and A. Antonio, J. Forensic Sci., 49, 364-370(2004).

11. Y.-Z. Liu and J. Yu, J. Chromatography A, 1125, 95103(2006) 